\title{
The effect of various physical exercise modes on perceived psychological stress
}

M K Magalhaes Das Neves, ${ }^{1}$ MPhil (Biokinetics); J M Loots, ${ }^{1}$ DSc (Physiol), DTech (Agric); R L van Niekerk, ${ }^{2}$ DLit et Phil (Psychology)

${ }^{1}$ Department of Sport and Movement Studies, Faculty of Health, University of Johannesburg, South Africa

${ }^{2}$ Department of Psychology, Faculty of Humanities, University of Johannesburg, South Africa

Corresponding author: R L van Niekerk(leonvn@uj.ac.za)

Background. Stress is an integral part of daily living, but chronic activation of the stress response without the ability to express the physical response results in overloading of the physiological and psychological systems.

Objective. To decrease perceived stress by means of one known and/or one relatively unknown physical activity, namely aerobic exercise and somatic awareness exercise.

Methods. This investigation made use of a quantitative, comparative, experimental research design over an 8-week period using pre- and post-tests. Participants were measured for psychological stress using a perceived stress scale. The participants were divided into those who were physically untrained and those who participated in exercise training programmes.

Results. There were five groups in total. The physically untrained individuals were divided into four groups: a somatic awareness exercise group ( $n=9)$; an aerobic exercise group $(n=15)$; a combination group taking part in both somatic awareness and aerobic exercise $(n=8)$; and a control group $(n=15)$. The 5th group contained individuals who participated in physical exercise prior to entering the programme; they were given somatic awareness exercise $(n=9)$ in addition to their existing physical exercises. An independent samples $t$-test revealed significant changes for perceived stress in the aerobic, somatic, combination and exercise groups, with a $95 \%$ confidence level in comparison with the control group.

Conclusion. Various physical modes such as aerobic exercise, somatic awareness training and a combination of the two may be used to decrease one's perceived stress in moderately to highly stressed individuals who are sedentary or who are physically active.

S Afr J SM 2014;26(4):104-108. DOI:10.7196/SAJSM.476

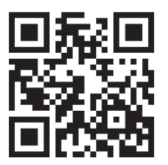

Stress is an integral part of the life of every human being, and is necessary for human functioning and adaptation. If the demands of life, in modern society for example, surpass the ability to cope, one will be in distress or in a negative stressful state. In order for a situation to be deemed as negatively stressful or not, one needs to perceive or appraise one's demands and ability to cope; this is defined as perceived stress. ${ }^{[1]}$ Chronic exposure to stress can be detrimental to one's physiological and psychological functioning, and result in chronic stress-related diseases. ${ }^{[2,3]}$ Worldwide, people manifest stressrelated diseases, and since excessive stress has been poorly managed, there have been increases in stress levels as high as $44 \%{ }^{[4]}$ In the working environment, South Africans have high perceived stress levels, with the greatest prevalence of stress among whites, females and individuals in the age group 20 - 30 years ${ }^{[5]}$ Annually, there can be as much as a R12 billion loss in South Africa (SA) due to stress-related absenteeism and low work productivity, which causes huge losses in economic profits and growth. ${ }^{[6]}$

It has also been found that SA individuals who have high levels of psychological stress do not partake in physical exercise, and engage in poor behavioural patterns (such as alcohol consumption, smoking and drug abuse) because of an inability to cope with the high levels of perceived stress. ${ }^{[7]}$ Poor behavioural patterns in conjunction with decreased physical activity levels can lead to increases in perceived stress and manifestation of chronic disease conditions, which are currently increasing rapidly in the SA population. ${ }^{[8]}$
The increased prevalence of stress-related disease indicates a need for improved stress management, such as the use of the psychological technique of cognitive behavioural therapy ${ }^{[9]}$ However, physiological techniques such as yoga ${ }^{[9]}$ and aerobic exercise ${ }^{[10]}$ have been shown to be just as effective in decreasing perceived stress as cognitive behavioural therapy. It is important to employ a physiological technique and not only a psychological technique, because the stress response is in itself physical ${ }^{[2,11]}$ and there needs to be a healthy physical outlet.

Although aerobic exercise is widely used to decrease perceived stress, it has also been shown to have no or little effect on perceived stress, ${ }^{[12]}$ and it is for this reason that the objective of the present study is to investigate the efficacy of somatic awareness exercise techniques (a different physiological mode) on decreasing perceived stress. (For a complete literature review on stress and somatic awareness, please contact the authors.) These techniques have been found to be effective in decreasing stress because they address both psychological and physiological aspects of the individual, and holistically integrate the self. $^{[13]}$

Somatic awareness techniques both implement and develop improved breathing, attention to the self, embodied presence, postural stability and quality of movement. ${ }^{[13]}$ This is achieved by teaching correct movement patterns, increasing someone's awareness of the acute and chronic physical manifestations of poor and correct movement patterns, and by doing so also increasing awareness of the self. $^{[13]}$ 
Table 1. Distribution of the age, BMI, gender and ethnicity of each group

\begin{tabular}{|c|c|c|c|c|c|c|c|}
\hline \multirow[b]{2}{*}{ Group } & \multirow[b]{2}{*}{ Mean age (years) } & \multirow[b]{2}{*}{ Mean BMI $\left(\mathrm{kg} / \mathrm{m}^{2}\right)$} & \multicolumn{2}{|c|}{ Gender, $n$} & \multicolumn{3}{|c|}{ Ethnicity, $n$} \\
\hline & & & Males & Females & White & Black & Indian \\
\hline Aerobic & 44 & 30.77 & 7 & 8 & 3 & 10 & 2 \\
\hline Somatic & 46 & 30.82 & 1 & 8 & 9 & 0 & 0 \\
\hline Combination & 37 & 23.86 & 1 & 7 & 6 & 0 & 2 \\
\hline Exercise & 31 & 25.52 & 0 & 9 & 8 & 0 & 1 \\
\hline Control & 38 & 25.15 & 4 & 11 & 13 & 2 & 0 \\
\hline
\end{tabular}

\section{Objective}

To investigate the efficacy of somatic awareness exercise on perceived stress, and to compare this with a known stress-reducing mode, namely aerobic exercise, in both trained and untrained individuals.

\section{Methods}

\section{Participants}

Fifty-six participants were recruited from corporate companies in the Johannesburg area. The volunteers completed an informed consent form, a medical screening form and a perceived stress scale (PSS). Subjects were excluded from the present study if their stress levels were low (PSS score $<10$ ), they were pregnant, and if they suffered from chronic illness and any absolute and relative contraindications to exercise according to the American College of Sports Medicine. ${ }^{[14]}$ Individuals who smoked were also excluded from the study, as smoking would have presented another variable that may have affected the study.

The mean age of the total sample was 41 (standard deviation (SD) 11, range 22 - 65) years. The total sample comprised 43 (77\%) females and $13(23 \%)$ males. The ethnicity of the total sample comprised white $(n=39,69.6 \%)$, Indian $(n=5,8.9 \%)$ and black $(n=12$, $21.4 \%)$ participants.

Most of the participants included in the study did not partake in regular physical exercise. These untrained (sedentary) participants were randomly assigned to either a control $(n=15)$, an aerobic exercise $(n=15)$, a somatic awareness $(n=9)$ or a combination (aerobic exercise and somatic awareness) $(n=8)$ group. Some subjects who volunteered to participate in the study were physically active prior to the study, and were placed in a separate exercise group $(n=9)$. The mean age, mean BMI, gender and ethnic distribution across the experimental and control groups are presented in Table 1. Both the Kolmogorov-Smirnov test for normality and Levene's test for homogeneity of variance were non-significant between the stress levels of the different groups, implying no statistical differences between the groups' stress levels. No statistical differences between the age and BMI of the groups were found.

\section{Research design}

The current investigation made use of a quantitative, comparative, experimental research design over an 8-week period, using pre- and post-tests. The methodological framework of the study is outlined in Table 2.

\section{Research instruments and method of assessment}

The research instrument used to measure perceived stress was the PSS, ${ }^{[1]}$ which has 10 items on a five-point Likert scale, with scores ranging from 0 to 40 . The PSS was used in the pretest, which took place 1 day before commencement of the sessions, and in the posttest, which took place 1 day after the last exercise session.

Normative scores on the test indicate that when a participant scores $<10$ on the PSS, they are experiencing minimal stress; a score of 10 - 20 indicates moderate stress, while a score of 20 - 30 indicates high stress and a score $>30$ indicates very high stress. The PSS was utilised because it takes into account the degree to which a person will perceive his/her life as stressful and how much stress is present in his/her life, rather than just measuring the physical response to a specific stressor. ${ }^{[1]}$ It is one of the most reliable and valid tests available for subjective stress appraisal and has a reliability score (Cronbach's alpha) of $0.72 .^{[15]}$

\section{The intervention protocol}

Prior to the intervention programme, all groups were informed that they had to participate in the study for 8 weeks, and that the required participation in the given intervention was a minimum of three times a week and a maximum of six times a week. ${ }^{[14]}$ This required 24 exercise sessions over the 8-week period. The subjects were allowed to exercise at a time convenient to them, because there is no marked difference in stress levels that is dependent on the time that one exercises. ${ }^{[1]}$

In the introductory sessions, all four intervention groups were shown the exercises by means of a DVD, which provided auditory and visual instructions. Each group had their own DVD with set instructions and the necessary exercise protocol. The control group did not receive any of these instructions mentioned, but were asked to continue with life as usual. After the introductory sessions, the participants were not supervised, but were monitored by means of weekly phone calls and emails. Participants in the intervention groups were also issued with an exercise diary to determine participant compliance, and to record their exercise sessions, duration, dates and overall mood.

\section{Aerobic exercise group}

The aerobic exercise protocol comprised a 5 - 10 min aerobic warm-up at a low intensity of 5 - 8 on the Borg Scale. The warm-up was followed by any moderate aerobic exercise of $10-12$ intensity on the Borg Scale for 20 - $30 \mathrm{~min},{ }^{[16]}$ as this has been shown to contribute most to stress relief. ${ }^{[11]}$ The cool-down was performed similarly to the warmup: $5-10 \mathrm{~min}$ at a low intensity of $5-8$ on the Borg Scale. ${ }^{[16]}$ Static 
Table 2. Methodological framework of the present study

\begin{tabular}{|c|c|c|c|c|}
\hline \multicolumn{5}{|l|}{ Sample } \\
\hline \multicolumn{5}{|l|}{56 participants } \\
\hline \multicolumn{5}{|c|}{ Ranked as moderately to highly stressed } \\
\hline \multicolumn{5}{|l|}{ From the Johannesburg area } \\
\hline \multicolumn{5}{|l|}{ Ages from 18 to 65} \\
\hline \multicolumn{5}{|c|}{ Subjects met inclusion criteria } \\
\hline \multicolumn{5}{|l|}{ Pretest } \\
\hline \multicolumn{5}{|c|}{ Retrospective health questionnaires and informed consent } \\
\hline \multicolumn{5}{|c|}{ Psychological screening: stress score } \\
\hline \multicolumn{5}{|c|}{ Subjects not exercising prior to study randomly assigned into four groups } \\
\hline \multicolumn{5}{|c|}{ Exercising subjects were assigned to an exercise group } \\
\hline \multicolumn{5}{|l|}{ Intervention protocols } \\
\hline Group 1: & Group 2: & Group 3: & Group 4: & Group 5: \\
\hline Aerobic & Somatic & Combination & Exercise & Control group \\
\hline Warm-up: & Warm-up: & Warm-up: & Warm-up: & No exercise \\
\hline Duration: 5 - $10 \mathrm{~min}$ & Duration: 5 - $10 \mathrm{~min}$ & Duration: 5 - $10 \mathrm{~min}$ & Duration: 5 - $10 \mathrm{~min}$ & \\
\hline $\begin{array}{l}\text { Intensity: Borg Scale } \\
\text { of } 5 \text { - } 8\end{array}$ & $\begin{array}{l}\text { Intensity: Borg Scale } \\
\text { of } 5-8\end{array}$ & $\begin{array}{l}\text { Intensity: Borg Scale } \\
\text { of } 5-8\end{array}$ & $\begin{array}{l}\text { Intensity: Borg Scale } \\
\text { of } 5-8\end{array}$ & \\
\hline Exercises: & Exercises: & Exercises: & Exercises: & \\
\hline Type: Aerobic & No aerobic exercise & Type: Aerobic & Continue with present & \\
\hline Duration: 20 - $30 \mathrm{~min}$ & & Duration: $20-30 \mathrm{~min}$ & exercise programme & \\
\hline \multirow[t]{3}{*}{$\begin{array}{l}\text { Intensity: Borg Scale of } \\
10-12\end{array}$} & & $\begin{array}{l}\text { Intensity: Borg Scale of } \\
10-12\end{array}$ & & \\
\hline & AND & AND & AND & \\
\hline & $\begin{array}{l}\text { Somatic awareness } \\
\text { exercises } \\
\text { (Lessons } 1-8)\end{array}$ & $\begin{array}{l}\text { Somatic awareness } \\
\text { exercises } \\
\text { (Lessons } 1-8 \text { ) }\end{array}$ & $\begin{array}{l}\text { Somatic awareness } \\
\text { exercises } \\
\text { (Lessons } 1-8)\end{array}$ & \\
\hline Cool down: & Cool down: & Cool down: & Cool down: & \\
\hline Duration: $5 \mathrm{~min}$ & Duration: 5 min & Duration: 5 min & Duration: 5 min & \\
\hline $\begin{array}{l}\text { Intensity: Borg Scale } \\
\text { of } 5-8\end{array}$ & $\begin{array}{l}\text { Intensity: Borg Scale } \\
\text { of } 5-8\end{array}$ & $\begin{array}{l}\text { Intensity: Borg Scale } \\
\text { of } 5 \text { - } 8\end{array}$ & $\begin{array}{l}\text { Intensity: Borg Scale } \\
\text { of } 5-8\end{array}$ & \\
\hline \multicolumn{5}{|l|}{ Post-test } \\
\hline \multicolumn{5}{|l|}{ Stress score } \\
\hline \multicolumn{5}{|l|}{ Statistics } \\
\hline \multicolumn{5}{|l|}{ Descriptive statistics } \\
\hline \multicolumn{5}{|l|}{ One-way ANOVA } \\
\hline \multicolumn{5}{|l|}{ Post-hoc comparisons } \\
\hline \multicolumn{5}{|l|}{ Paired sample $t$-test } \\
\hline Effect size & & & & \\
\hline
\end{tabular}

ANOVA $=$ analysis of variance.

stretching was performed after cool-down, and was chosen because stretching has been shown to decrease the stress response and induce a physiological and psychological relaxation response. ${ }^{[10]}$ The stretches included the stretching of gross musculature (lower back, hamstrings, neural hamstrings, quadriceps, hip flexors, trunk extensors, obliques, quadratus lumborum, groin, calves, triceps and neck) for $30 \mathrm{~s}$, with each stretch repeated three times. Prior to the intervention programme, the stretching instructions and guidelines ${ }^{[14,16]}$ were discussed with, and demonstrated to, the participants, with emphasis on correct breathing, limb placement and range of motion.

\section{Somatic awareness group}

The main body of work and the structure of the intervention were taken from Hanna. ${ }^{[13]}$ Somatic awareness exercises have, as their 
Table 3. Mean PSS pretest and post-test scores, mean differences, significance and effect size of all five groups

\begin{tabular}{lllllll}
\hline Variable & Group & Pretest, mean $(\mathrm{SD})$ & Post-test, mean $(\mathrm{SD})$ & Mean pre- and post-test difference & $\boldsymbol{p}$-value & Effect size $\left(\right.$ eta $\left.{ }^{2}\right)$ \\
\hline PSS & Aerobic & $18.80(5.60)$ & $14.20(6.64)$ & -4.60 & 0.02 & 0.33 \\
& Somatic & $24.11(3.44)$ & $15.44(5.46)$ & -8.67 & 0.01 & 0.76 \\
Combination & $23.38(6.35)$ & $16.63(3.62)$ & -6.75 & 0.01 & 0.76 \\
& Exercise & $25.44(4.04)$ & $18.33(5.17)$ & -7.11 & 0.01 & 0.75 \\
& Control & $19.47(5.68)$ & $18.87(5.83)$ & -0.60 & 0.66 & -
\end{tabular}

primary goal, an increase in somatic awareness in the individual. This increased awareness is attained by teaching correct movement patterns in various parts of the body. The movements are all slow and gentle, with the emphasis on being totally aware of the proprioceptive input from each and every part of the body. This then increases the ability of participants to become aware of their own movements and posture, and make appropriate corrections where and when required. It should be noted that none of these lessons included any form of stretching. The somatic awareness training had to be done three times a week for a total of 8 weeks. The sessions were divided into eight lessons. Each lesson focused on increasing awareness and releasing muscle tension in a particular group of muscles. ${ }^{[13]}$

- Lesson 1: Extensor back muscles. This lesson focused on increasing awareness of the lower back muscles and decreasing lumbar extensor tension by increasing movement in the hips.

- Lesson 2: Flexor muscles of stomach. The lesson built on lesson 1, where the back extensors were integrated with the abdominal and anterior musculature. The participant was taught to integrate the muscles and the movements using the hip muscles, allowing for the agonists and antagonists to work together.

- Lesson 3: Muscles of the waist. This lesson concentrated on the waist muscles, such as the internal and external obliques, and the quadratus lumborum. The point of the lesson was to increase the muscle length at the waist and decrease the feeling of tightness in the obliques and the quadratus lumborum.

- Lesson 4: Controlling muscles involved in trunk rotation. This lesson took advantage of the growing sensitivity and control attained in lessons 1 - 3. The main movements were rotational movements of the trunk muscles (quadratus lumborum, abdominals, back extensors), with attention to the elongation (lengthening) and awareness of the function of the trunk muscles.

- Lesson 5: Hip and leg muscles. In this lesson, the participant was taught how to free the muscles of the trunk, hips, legs and feet. The basics of locomotion and the freeing up of movement along the entire lower body were analysed.

- Lesson 6: Neck and shoulders. This lesson focused on increasing upper body awareness, freeing up the ribs, chest, shoulders and neck, and increasing the movements of the upper body.

- Lesson 7: Breathing. The focus of this lesson was on breathing, specifically breathing done with the diaphragm.

- Lesson 8: Walking. This lesson built on what had been achieved in lessons 1 - 7. It taught walking with proper trunk, spinal and shoulder rotation, as well as the rotation experienced by the arms and legs. It emphasised the contralateral walking pattern, and making walking effortless and graceful.

\section{Combination group}

The combination group had to combine both the aerobic exercise and the somatic awareness exercise in the same session on the same day (they were not allowed to pick only one). They were required to do both exercises a minimum of three times a week for a period of 8 weeks. On the days they chose to do aerobic exercise, they had to undergo the aerobic exercise protocol described above as well as the somatic awareness exercise (see Table 2).

\section{Exercise group}

The exercise group was instructed to continue with their aerobic programmes, but somatic awareness exercise was added. They had to complete aerobic exercise and the somatic awareness exercise at least three times a week for 8 weeks (see Table 2).

\section{Ethical considerations}

The present study was granted ethical approval by the ethical committee of the University of Johannesburg prior to its commencement. A letter of information was issued to each participant, including an informed consent form.

\section{Data analysis}

Frequency and descriptive statistics were conducted to describe the sample. The mean scores and SDs for both the pre- and post-test of the PSS were calculated. Statistical significance was set at $p<0.05$ for the study. Normality was determined by the Kolmogorov-Smirnov test and the Shapiro-Wilk test. A normal distribution was found for all PSS pre- and post-test measures in all the intervention groups, except for the post-test of the somatic group. Paired-sample $t$-tests were done to determine within-group changes in PSS after the interventions, and the effect size was determined by calculating the eta ${ }^{2}$ statistic. Guidelines for interpretation of the effect size were: $0.01-0.06$ : small effect; 0.07 - 0.14: moderate effect; and $>0.14$ : large effect. A one-way analysis of variance (ANOVA) and post-hoc tests (Dunnett T3) were conducted to determine group differences.

\section{Results}

The results of the PSS scores and the distribution of the demographics of each group are shown in Table 3.

After statistical analysis, there was no statistically significant difference in compliance between the somatic, combination and 
exercise groups. However, while the aerobic exercise group did have a statistically significant increase in compliance, it did not positively affect objective stress measures, nor did less participation in aerobic exercise negatively affect the objective stress measures. Thus, one may assume that compliance in the aerobic exercise group did not affect the current study.

\section{Discussion}

There were statistically significant decreases in the perceived stress of all the experimental groups, and although the perceived stress results for the control group decreased, this decrease was not statistically significant.

A small effect size was found for the aerobic group, indicating that aerobic exercise was only slightly effective in decreasing perceived stress. A key finding in this study, however, was the large effect sizes found with the experimental groups that did somatic awareness exercise (somatic, combination and exercise groups); the perceived stress score decreased significantly in these groups. The large effect size of somatic awareness exercise may be due to its properties, which are not present in aerobic exercise. Although it may seem that aerobic exercise and the use of stretching promote somatic awareness, they do not, as they do not focus on somatic awareness principles such as teaching of motor movements, improved kinaesthetic awareness, ${ }^{[17]}$ sensory processing, ${ }^{[18]}$ internal feedback and internal attention. ${ }^{[19]}$ Thus, by allowing improved feedback processing, stressor perception and emotional regulation, there is an improvement (decrease) in perceived stress. ${ }^{[20]}$

The combination group had a statistically significant decrease in perceived stress scores, and a larger effect size than the group that participated in aerobic exercise only, because the combination of two or more modes has been shown to be more effective than one alone. The exercise group also had a statistically significant decrease with a large effect size. ${ }^{[17]}$ It is interesting to note that while the participants in this group were exercising, they still had high levels of perceived stress. However, regardless of what their exercise modalities were, the addition of the somatic awareness exercise brought about the large effect size and the decrements in the perceived stress score. These results indicate that somatic awareness exercise is a more effective physical mode than the other modes in this study than the other modes in this study in reducing perceived stress, whether it is used alone or in conjunction with other cardiovascular modes, in both trained and untrained individuals.

Since perceived stress can be reduced by various physical modalities, the results of this study indicate how beneficial it could be to add somatic awareness training (which includes combinations of breathing, mindfulness, movement and inner sensing) to any physical mode in order to reduce perceived stress in individuals who are experiencing moderate to high stress levels.

There are a few recommendations according to the limitations of the study. Firstly, the sample could be larger, with a more evenly distributed gender and ethnicity profile. Secondly, the specific protocol utilised in the study could not be found in other studies. In order to test its reliability, one should repeat the study and compare it with other mindfulness techniques such as yoga, tai chi and mindfulnessbased stress reduction.

\section{Conclusion}

On the basis of the results of the present study, it may be concluded that various physical modes such as aerobic exercise, somatic awareness training and the combination of the two may be used to decrease perceived stress levels in moderately to highly stressed individuals who are sedentary or who are physically active. However, the results suggest that somatic awareness exercise is a more effective physical mode than the other modes in this study in reducing perceived stress, whether it is used alone or in conjunction with other cardiovascular modes, in both trained and untrained individuals.

\section{References}

1. Cohen S, Kamarck T, Mermelstien R. A global measure of perceived stress. J Health Soc Behav 1983;24(4):385-396.

2. Chrousos GP. Stress and disorders of the stress system. Nat Rev Endocrinol 2009;5(7):374-381. [http://dx.doi.org/10.1038/nrendo.2009.106]

3. Cohen S, Janicki-Deverts D, Miller GE. Psychological stress and disease. JAMA 2007;198(14):1685-1687. [http://dx.doi.org/10.1001/jama.298.14.1685]

4. Health and Safety Executive. Stress and Psychological Disorders, 2011. http://www. hse.gov.uk/statistics/causdis/stress/stress.pdf (accessed 26 September 2012).

5. Coopmans JWM. Stress Related Causes of Presenteeism amongst South African Managers. MPhil dissertation. Pretoria: University of Pretoria, 2007.

6. Lilford N. Absenteeism Reaches New Heights in South Africa, 2010. http:// www.hrfuture.net/wellness/absenteeism-reaches-new-heights-in-south-africa php? Itemid= 183 (accessed 3 October 2012).

7. Brook DW, Rubenstone E, Zhang C, Morojele NK, Brook JS. Environmental stressors, low well-being, smoking, and alcohol use among South African adolescents. Soc Sci Med 2011;72(9):1447-1453. [http://dx.doi.org/10.1016/2011.02.041]

8. Westaway MS. The impact of chronic diseases on the health and well-being of South Africans in early and later old age. Arch Gerontol Geriatr 2010;50(2):213-221. [http:// dx.doi.org/10.1016/j.archger.2009.03.012]

9. Granath J, Ingvarsson S, von Thiele U, Lundberg U. Stress management: A randomised study of cognitive behavioural therapy and yoga. Cogn Beav Ther 2006;35(1):3-10. [http://dx.doi.org/10.1080/16506070500401292]

10. Bond DS, Lyle RM, Tappe MK, Seehafer RS, D'Zurilla TJ. Moderate aerobic exercise, t'ai chi, and social problem-solving ability in relation to psychological stress. Int Stress Manag 2002;9(4):329-343. [http://dx.doi.org/10.1023/A:1019934417236]

11. Cox RH. Sports Psychology Concepts and Applications. 5th edition. New York: McGraw-Hill, 2002.

12. Hansen CJ, Stevens LC, Richard CJ. Exercise duration and mood state: How much is enough to feel better? Health Psychol 2001;20(4):267-275. [http://dx.doi. org/10.1037//0278-6133.20.4.267]

13. Hanna T. The Body of Life: Creating New Pathways for Sensory Awareness and Fluid Movement. Rochester, USA: Healing Arts Press, 1993.

14. American College of Sports Medicine. Pre-exercise evaluations. In: Whaley $\mathrm{MH}$ Brubaker PH, Otto RM, eds. ACSM's Guidelines for Exercise Testing and Prescription. 7th ed. Philadelphia: Lippincott Williams \& Wilkins, 2006:79-173.

15. Benham G. The highly sensitive person: Stress and physical symptom reports. Pers Individ Dif 2006;40(7):1433-1440. [http://dx.doi.org/10.1016/j.paid.2005.11.021]

16. Baechle TR, Earle RW. Essentials of Strength Training and Conditioning. Champaign, USA: Human Kinetics, 2000.

17. Alexander FM. Constructive Conscious Control of the Individual. London: Victor Gollancz Ltd, 1987.

18. Kilpatrick LA, Suyenobu BY, Smith SR, et al. Impact of mindfulness-based stress reduction training on intrinsic brain connectivity. Neuroimage 2011;56(1):290-298 [http://dx.doi.org/10.1016/j.neuroimage.2011.02.034]

19. Kerr GA, Kotynia F, Kolt G. Feldenkrais awareness through movement and state anxiety J Bodyw Mov Ther 2002;6(2):102-107. [http://dx.doi.org/10.1054/jbmt.2001.0274]

20. Dobkin PL. Mindfulness-based stress reduction: What processes are at work? Complement Ther Clin Pract 2008;14(1):8-16. [http://dx.doi.org/10.1016/j.ctcp.2007.09.004] 